\title{
La dieta y la fauna de parásitos metazoos del torito Bovichthys chilensis Regan 1914 (Pisces: Bovichthydae) en la costa de Chile centro-sur: variaciones geográficas y ontogenéticas
}

\author{
Diet and metazoan parasite fauna of the thornfish Bovichthys chilensis Regan 1914 \\ (Pisces: Bovichthydae) on the coast of central-south Chile: geographical and \\ ontogenetic variations
}

GABRIELA MUÑOZ, VERÓNICA VALDEBENITO \& MARIO GEORGE-NASCIMENTO

Facultad de Ciencias, Universidad Católica de la Santísima Concepción, Casilla 297, Concepción, Chile; e-mail: mgeorgen@ucsc.cl

\begin{abstract}
RESUMEN
Conocer qué, cuánto, cuándo y dónde comen y viven los hospedadores permitiría complementar los estudios parasitarios, ya que la transmisión de los endoparásitos está estrechamente ligada a la dieta, y la de los ectoparásitos al uso del hábitat. Por esto, se describen y comparan la composición y características cuantitativas de la dieta y de las infracomunidades de parásitos metazoos del torito Bovichthys chilensis con datos obtenidos de 108 ejemplares juveniles recolectados desde la zona intermareal de cuatro localidades de la costa de Chile (entre $33^{\circ}$ y $40^{\circ} \mathrm{S}$ ), y de 14 adultos recolectados desde el submareal somero de una quinta localidad $\left(36^{\circ} \mathrm{S}\right)$, y se discute los resultados a la luz de los cambios ontogenéticos en el nicho de este huésped. Cerca del $70 \%$ de los ejemplares tenía contenido alimentario, en el que se distinguieron 25 ítems presa, de los cuales sólo uno era compartido entre juveniles y adultos. La dieta de los toritos juveniles estuvo compuesta principalmente por anfípodos y la de los adultos por crustáceos decápodos. Cerca de un $40 \%$ de los toritos albergaba un total de 624 parásitos en los que se reconocieron 16 taxa, y sólo cuatro eran compartidos entre juveniles y adultos. En los toritos juveniles muestreados en las cuatro localidades había baja y similar intensidad total, riqueza y diversidad parasitarias, y variaciones geográficas significativas en la prevalencia total, composición de la dieta y de las infracomunidades de parásitos. La falta de una relación clara entre la composición de la dieta y del parasitismo en los toritos juveniles puede deberse a que las parasitosis son necesariamente recientes, y a que pueden haber grandes diferencias en el tiempo de residencia de presas y parásitos en el tracto digestivo. En los toritos adultos hubo mayor prevalencia, intensidad y diversidad de parásitos que en los juveniles de una localidad cercana. Se requieren más estudios, en especial en la variación geográfica del parasitismo de los toritos adultos, para dilucidar si en esta especie de hospedador el eje ontogenético es o no más importante que el geográfico para explicar las variaciones de la fauna parasitaria.
\end{abstract}

Palabras clave: dieta, parásitos, ontogenia, Bovichthys chilensis, Chile.

\section{ABSTRACT}

Knowledge on what, how much, when and where hosts eat and live should help to understand the changes that occur in parasite communities because transmission of endoparasites is tightly linked to diet composition as well as ectoparasites are to the use that hosts make of the habitat. Thus, geographical and ontogenetic variations in diet and parasite fauna of the thornfish Bovichthys chilensis are described, and discussed taking into account the ontogenetic shifts in this host's niche. A total 122 individuals: 108 juveniles collected from the intertidal zone at four localities along the south-central coast of Chile (between $33^{\circ}$ and $40^{\circ} \mathrm{S}$ ), and 14 adults collected from the shallow subtidal at a fifth locality $\left(36^{\circ} \mathrm{S}\right.$ ), were examined for food contents and parasite infracommunities. Food items were encountered in almost $70 \%$ of fish examined with 25 prey items being recognized. Only one of the prey items was found in both juvenile and adult fish. The diet of juvenile fish consisted mainly of amphipods and while that of adults was mainly composed of crustacean decapods. Almost $40 \%$ of fish examined harbored parasites with a total of 624 individuals and belonging to 16 taxa being identified, four of which were shared between juvenile and adult fish. Prevalence, total intensity, richness, and diversity of parasites was similarly low among juvenile fish sampled at four different localities, while significant differences in total prevalence, dietary and parasite infracommunity composition among localities were found. Absence of a clear relationship among juvenile fish between the composition of the diet and of infracommunities may be due to the fact that parasitoses are necessarily recent. Adult hosts had infracommunities with greater total prevalence, total intensity, taxonomic richness and diversity than juveniles sampled at a nearby locality. All these observations could be associated to ontogenetic shifts in diet and concomitant shifts in parasite fauna. Further studies are required, especially on geographical variations of the parasitism among adult thornfish to elucidate whether the host ontogenetic axis could be more important than the geographical in this species, when attempting to explain sources variations in the parasite fauna.

Key words: diet, parasites, host ontogeny, Bovichthys chilensis, Chile. 


\section{INTRODUCCIÓN}

El conocimiento de la ecología de una especie considera el uso del hábitat, la dieta, y los tiempos de actividad, entre otras variables. Todas ellas pueden variar a lo largo de la ontogenia, o por el sexo, y por ende afectar la composición y otras características de su fauna parasitaria. Por esto, determinar qué, cuánto, cuándo y dónde comen los hospedadores permitiría complementar los estudios parasitarios, ya que la transmisión de los endoparásitos está estrechamente ligada a la dieta, es decir, a través de presas que funcionan como hospedadores intermediarios para los parásitos (Williams \& Jones 1994). A pesar de esta relación funcional entre dieta y endoparasitismo, son escasos los estudios que examinan conjuntamente ambos aspectos (Riffo \& GeorgeNascimento 1992, Valtonen \& Julkunen 1995, Morand et al. 2000). Los ectoparásitos, en cambio, se relacionan más con el uso del hábitat, ya que se transmiten principalmente por contacto directo (Rohde 1984).

En Chile se ha registrado la dieta de algunos peces de la zona intermareal (ver Quijada \& Cáceres 2000), así como los factores que la afectarían, tales como la disponibilidad y selectividad del alimento (Ojeda \& Muñoz 1999), la ontogenia, el origen geográfico (Muñoz \& Ojeda 1998, 2000) y la contaminación (Fariña et al. 2000). Son pocos los estudios en parasitismo de peces intermareales en el mundo y menos aún los que relacionan dieta y parasitismo (Zander et al.1999, Aldana et al. 2002). Uno de ellos muestra que la relación entre los cambios geográficos y ontogenéticos de la dieta y del parasitismo no se corresponden nítidamente en dos especies de peces: Graus nigra y Girella laevifrons (Aldana et al. 2002).

En este estudio se describen la dieta y la parasitofauna del torito Bovichthys chilensis Regan, 1914, y se las compara entre localidades geográficas, y entre ejemplares juveniles y adultos. El objetivo es explorar las relaciones entre la composición de la dieta, el uso del hábitat y la composición de la fauna de parásitos. El torito $B$. chilensis es un pez que se distribuye en Chile desde Los Molles (32 $14^{\prime}$ 'S, Muñoz \& Ojeda 1997) hasta Valdivia (3950' S, Moreno \& Castilla 1975), y de la que no existen registros parasitológicos. Un análisis más actualizado y exhaustivo de la distribución geográfica de $B$. chilensis es el de Ojeda et al. (2000). Sólo los juveniles habitan en el intermareal rocoso, y se desplazan al submareal en donde llegan a ser adultos, por lo que son peces transitorios de las pozas intermareales (Muñoz \& Ojeda 1998). Las presas principales del torito son los anfípodos y los crustáceos decápodos, habiendo diferencias claras a través de la ontogenia (Muñoz \& Ojeda 1998). En base a estos cambios ontogenéticos en el uso del hábitat y de la composición de la dieta que experimenta el torito, predecimos grandes diferencias en la composición de las comunidades de parásitos en ejemplares juveniles y adultos muestreados en localidades vecinas. La discusión considera algunas condiciones en las que se puede esperar una correlación entre la composición de la dieta y de la fauna parasitaria de un hospedador.

\section{MATERIALES Y MÉTODOS}

Se capturaron 122 ejemplares de B. chilensis desde 5 localidades de la costa de Chile, entre febrero de 1998 y mayo de 1999. Los ejemplares entre 4 y $18 \mathrm{~cm}$ de longitud total fueron considerados juveniles (ver Muñoz \& Ojeda 1998), y fueron obtenidos desde pozas del intermareal rocoso mediante el uso de redes de mano. De éstos, 24 ejemplares provenían de Isla Negra $\left(33^{\circ} 25^{\prime} \mathrm{S}\right), 35$ de Burca ( $\left.36^{\circ} 32^{\prime} \mathrm{S}\right), 28$ de Los Bagres (36 $\left.37^{\circ} \mathrm{S}\right)$ y 21 de Pucatrihue (40 $30^{\prime}$ S). En Chome (36 $46^{\circ}$ ' S) se obtuvieron 14 ejemplares adultos (los que sobrepasaban los $18 \mathrm{~cm}$ ), que fueron recolectados desde el submareal somero, hasta $6 \mathrm{~m}$ de profundidad, mediante pesca con red de arrastre. Una vez capturados, cada ejemplar fue puesto en una bolsa plástica, y preservado a $-20{ }^{\circ} \mathrm{C}$ para su posterior examen.

La determinación taxonómica de los peces se realizó de acuerdo a Mann (1954). Cada ejemplar fue medido en su longitud total con $0,1 \mathrm{~cm}$ de sensibilidad. De cada ejemplar se separó y tamizó el contenido estomacal en un tamiz de $0,35 \mathrm{~mm}$ de luz a fin de obtener una mejor individualización de las presas macroscópicas. La resolución taxonómica en las presas no fue la misma para todos los taxa, y dependió en parte de su grado de digestión. La determinación taxonómica de las presas se llevó a cabo consultando una colección de referencia. Las presas de menor tamaño por lo general estaban más digeridas y fue más difícil identificarlas a nivel de especie. Los ítems presa fueron contabilizados y se determinó su peso (g), para luego obtener los siguientes descriptores cuantitativos propuestos por Hyslop (1980): el porcentaje del número total de las presas $(\% \mathrm{~N})$, porcentaje del peso total en los contenidos estomacales $(\% \mathrm{P})$, y la frecuencia porcentual de ocurrencia, definida como el número de veces en que el ítem ocurrió en el total de estómagos con contenido estomacal $(\% \mathrm{~F}$, ver totales al pie de la Tabla 2$)$. 
La recolección de parásitos se realizó bajo microscopio estereoscópico, en la piel, branquias, cavidad celomática, tracto digestivo y gónadas. Los parásitos fueron preservados en formalina al $10 \%$. La determinación taxonómica de los parásitos se llevó a cabo consultando a Kabata (1979) para los copépodos, a Yamaguti (1971) para los digeneos, a Zdzitowiecki (1991) y Muñoz \& George-Nascimento (2002) para los acantocéfalos.

En las muestras de cada localidad se calculó la prevalencia y abundancia de cada taxon parasitario, y los siguientes descriptores comunitarios: prevalencia total (\% de hospedadores parasitados en la muestra), abundancia total (número de parásitos por hospedador examinado), intensidad total (número de parásitos por hospedador parasitado), riqueza y diversidad infracomunitarias (índice de Brillouin, ver Bush et al. 1997), estas tres últimas calculadas con los ejemplares parasitados (Magurran 1988).

La longitud total de los ejemplares juveniles, así como la intensidad total, la riqueza y la diversidad infracomunitarias fueron comparadas entre localidades mediante la prueba de Kruskal-Wallis. Para comparar la prevalencia total entre pares de localidades se utilizaron pruebas de Chi-cuadrado (Siegel \& Castellan 1988).

El análisis de las variaciones de la composición de la dieta y de la fauna parasitaria contempló dos etapas. Primero se realizó una ordenación de la matriz de presencia - ausencia de los 25 ítems dietarios en los 89 estómagos con contenido, con un análisis de correspondencia con efecto de arco corregido (DCA). El mismo método, pero con los datos de abundancia relativa, fue usado para la ordenación de 46 infracomunidades y 9 taxa parasitarios. De la ordenación de las infracomunidades se excluyó a los taxa que poseían menos de 3 ocurrencias en el total de datos (Philometra sp., Caligus sp., P. moyanoi, Spiruridae gen. sp., Contracaecum sp., y H. magellanicus ocurrieron sólo una vez en las muestras y no fueron considerados en la ordenación de las infracomunidades). Este criterio fue adoptado porque las especies muy raras modifican en demasía los puntajes de las ordenaciones (Jongman et al. 1995), aunque no fue aplicado a las presas (ver Discusión).

En una segunda etapa, los puntajes de las ordenaciones fueron comparados entre toritos juveniles de distintas localidades, y entre juveniles y adultos de localidades cercanas, mediante análisis de la varianza no paramétricos de una vía (Zar 1996). La inclusión de infracomunidades sólo de localidades vecinas en la comparación entre estados ontogenéticos persiguió minimizar el potencial efecto de las variaciones geográficas. Sin embargo, por el escaso parasitismo total de una de las localidaes (Burca), esta comparación se realizó sólo entre dos localidades (Los Bagres (juveniles) y Chome (adultos)).

\section{RESULTADOS}

La longitud total de los toritos juveniles fue significativamente distinta entre las cuatro localidades muestreadas, a pesar del estrecho rango abarcado (entre 4,5-8,0 cm, aproximación a Chi-cuadrado de la prueba de Kruskal- Wallis: $\chi^{2}=10,1$; 3 g.1.; $\mathrm{P}<0,02$; Tabla 1). Sólo los ejemplares muestreados en Isla Negra y Pucatrihue no mostraron diferencias en longitud total (prueba a posteriori de Dunnet).

Ochenta y nueve de los 122 toritos presentaron contenido estomacal (72,9\%). En ellos se recolectaron 651 individuos presa con un peso total de $42,5 \mathrm{~g}$ y 161 ocurrencias, en las que se distinguieron 25 taxa ( $\sin$ considerar los restos de crustáceos decápodos y de peces), 14 de los cuales ocurrieron sólo en los juveniles. Mysidacea fue el único ítem dietario compartido entre toritos juveniles y adultos (Tabla 2). Los juveniles se alimentaban principalmente de anfípodos, aunque en los ejemplares recolectados en Burca, las presas más importantes fueron larvas de insectos (Tabla 2). La dieta de los toritos adultos estuvo compuesta principalmente por crustáceos decápodos, siendo Petrolisthes desmarestii la presa de mayor tamaño, frecuencia de ocurrencia y representación numérica (Tabla 2).

Las variaciones geográficas de la composición de la dieta de los toritos juveniles fueron evaluadas en el segundo eje de la ordenación (autovalor $=$

TABLA 1

Longitud total promedio $(\mathrm{cm})$ de 122 ejemplares de Bovichthys chilensis

recolectados en cinco localidades de la costa

de Chile; desviación estándar (DE), $\mathrm{n}=$ número de ejemplares muestreados

Mean total body length (cm) of 122 Bovichthys chilensis specimens collected at five localities along the coast of Chile; standard deviation (DE), $\mathrm{n}=$ number of specimens examined

\begin{tabular}{llll}
\hline & Localidad & $\mathrm{n}$ & $\begin{array}{c}\text { Longitud } \\
\text { total (DE) }\end{array}$ \\
\hline Juveniles & & & \\
& Isla Negra & 24 & $6,8(1,05)$ \\
& Burca & 35 & $5,3(0,46)$ \\
& Los Bagres & 28 & $5,9(0,74)$ \\
Adultos & Pucatrihue & 21 & $6,0(0,78)$ \\
& Chome & 14 & $29,7(1,39)$ \\
\hline
\end{tabular}




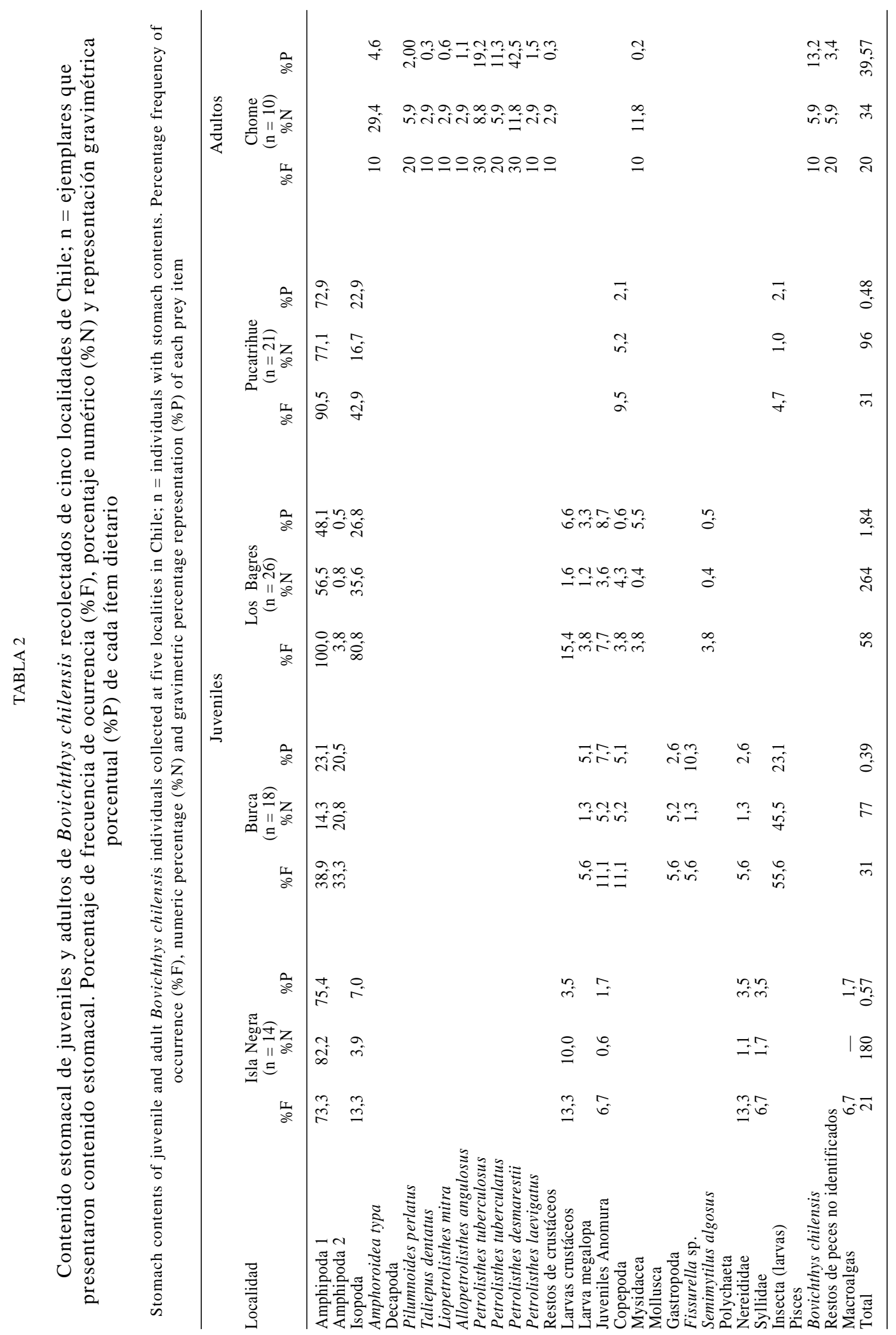


0,96), ya que el primer eje sólo separaba a juveniles de adultos (autovalor $=1$ ). En este segundo eje del DCA, un $59 \%$ de la variación dietaria de los juveniles estuvo explicada por la localidad de muestreo $\left(\mathrm{F}_{3,74}=36,1 ; \mathrm{P}=0,0001\right.$; Tabla 2, Fig. 1A). Estas diferencias se debían exclusivamente a que en la muestra de Burca había una gran representación de larvas de insecto, haciéndola distinta a las demás muestras (prueba a posteriori GT-2; P< 0,05). Esto se señala en la Fig. 1A en que la dieta de los ejemplares de Burca guardan diferencias de hasta cerca de seis desviaciones estándar con la de los de Isla Negra, Los Bagres y Pucatrihue.
La diferencia dietaria entre juveniles y adultos muestreados en localidades vecinas (Burca, Los Bagres y Chome) era de tal magnitud, que el $67 \%$ de la variación en el segundo eje del DCA $\left(\mathrm{F}_{2,39}=\right.$ $40,1 ; \mathrm{P}<0,001)$ estuvo explicada por el estado ontogenético del hospedador. La extensión del segundo eje del DCA de alrededor de 14 desviaciones estándar indica la casi total diferencia dietaria entre estados ontogenéticos (Fig. 1B).

El 39,3\% de los 122 ejemplares examinados albergaba al menos un taxón parasitario. En el total de 48 hospedadores parasitados se recolectaron 624 individuos parásitos pertenecientes a 16 taxa,

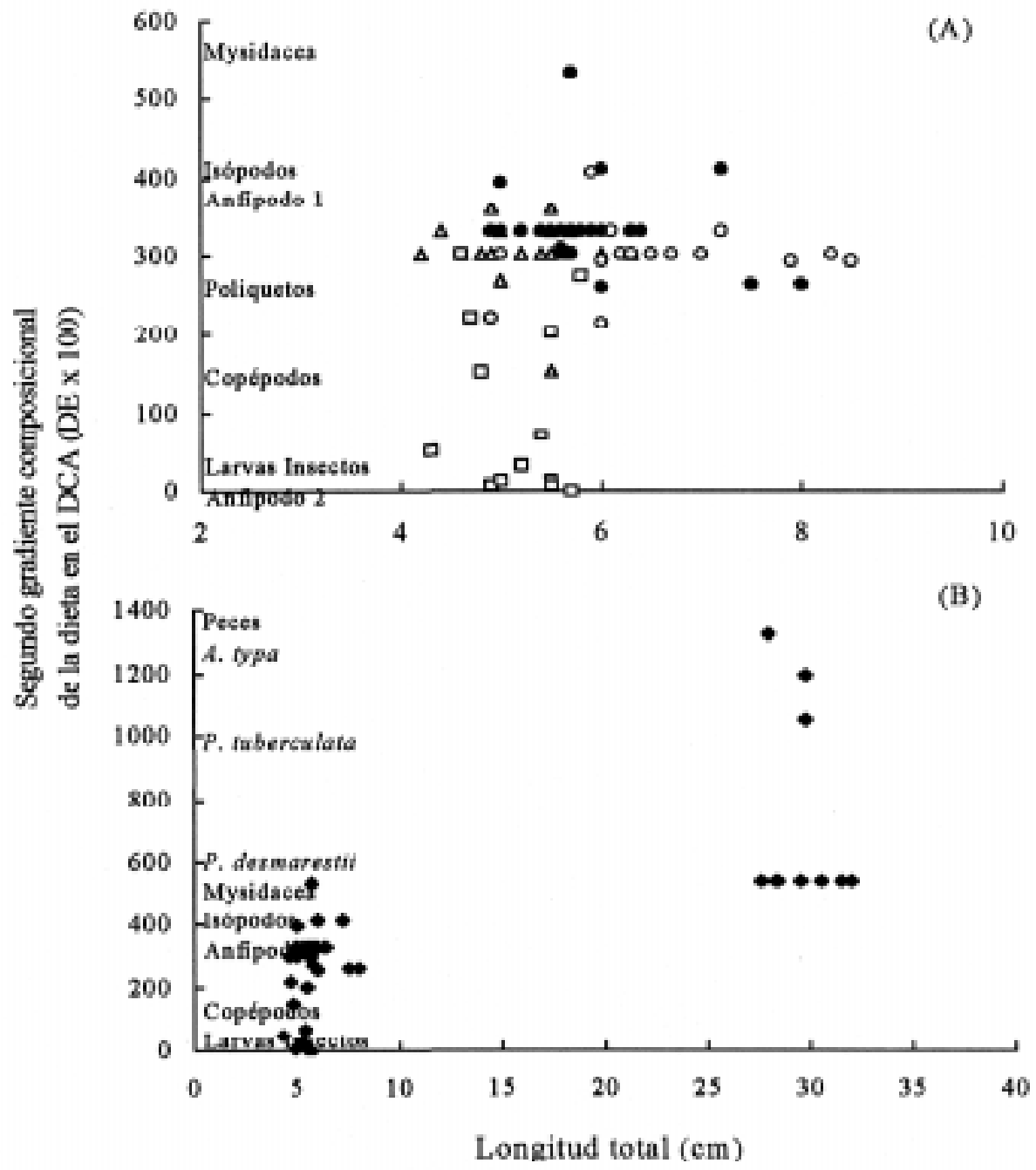

Fig. 1: Relación entre el gradiente composicional de la dieta (puntajes en el segundo eje del DCA) y la longitud total de B. chilensis en: (A) juveniles de cuatro localidades geográficas (Isla Negra = círculos blancos, Burca $=$ cuadrados blancos, Los Bagres = círculos negros, Pucatrihue $=$ triángulos blancos), $(\mathrm{B})$ juveniles y adultos de localidades vecinas del litoral centro-sur de Chile ( $\left.36^{\circ} \mathrm{S}\right)$. Los nombres de los ítems dietarios se indican frente a su coordenada en el eje.

Relationship between dietary compositional gradient (scores on second DCA axis) and total body length of B. chilensis: (A) juveniles from four localities (Isla Negra $=$ white circles, Burca $=$ white squares, Los Bagres $=$ black circles, Pucatrihue $=$ white triangles $)$, and (B) juveniles and adults from three nearby littoral localities in central-south Chile $\left(36^{\circ}\right.$ $\mathrm{S})$. Names of food items are shown in front of their coordinate in that axis. 


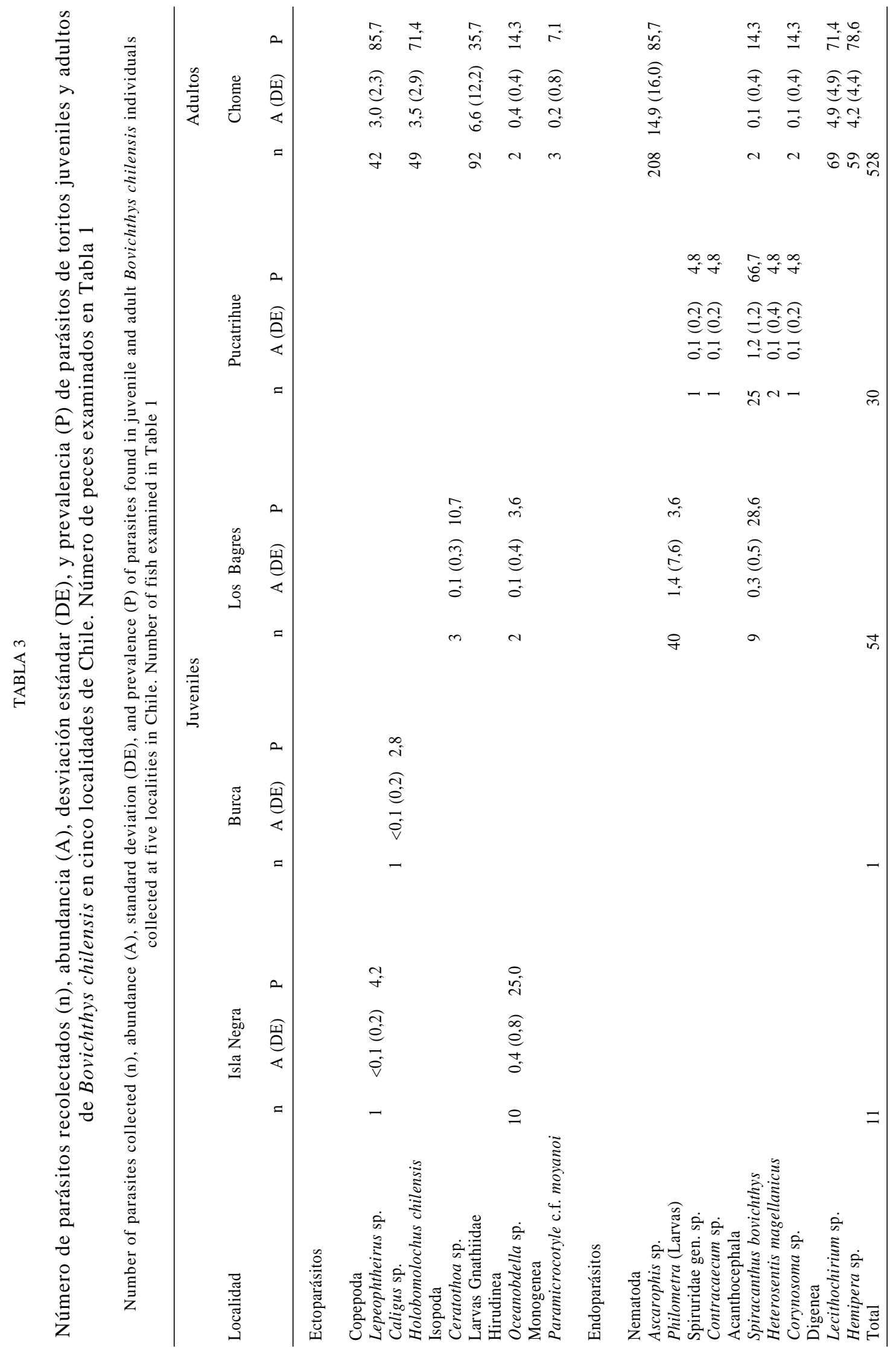


de los cuales siete eran ectoparásitos y nueve eran endoparásitos (Tabla 3). La ordenación de 46 infracomunidades y 10 taxa mostró un autovalor de 0,92 en el primer eje del DCA (Fig. 2), lo que significa que hay una correlación cercana a 0,96 entre los puntajes de las especies (parásitos) y de los sitios (hospedadores, ver Pielou 1984). La extensión de cerca de seis desviaciones estándar en este primer eje indica que hubo infracomunidades totalmente distintas en composición (Fig. 2).

Se observaron diferencias geográficas significativas en la composición de la fauna parasitaria de los toritos juveniles. Las infracomunidades examinadas en hospedadores de Isla Negra eran distintas de las de Los Bagres y Pucatrihue, por la mayor presencia de la sanguijuela Oceanobdella sp., (Burca no fue incluída). El $56 \%$ de la variación en composición de las infracomunidades de los toritos juveniles estuvo explicado por la localidad de muestreo $\left(\mathrm{F}_{2,29}=18,2 ; \mathrm{P}<0,001\right.$; Fig. 2A). Sin embargo, no se encontraron diferencias entre localidades en intensidad total (aproximación a Chi-cuadrado de la prueba de KruskalWallis: $\left.\chi^{2}=2,8\right)$, riqueza $\left(\chi^{2}=1,8\right)$, y diversidad de parásitos $\left(\chi^{2}=1,7\right.$, todos con 3 g.l.; $0,40<\mathrm{P}<$ 0,70 ; Tabla 4). En general, también se observaron diferencias geográficas significativas en la prevalencia total del parasitismo entre juveniles (excepto entre los muestreados en Isla Negra y Los Bagres, Tabla 4 y 5).

Cuatro de los 16 taxa eran compartidos entre toritos juveniles y adultos: Lepeophtheirus sp.,

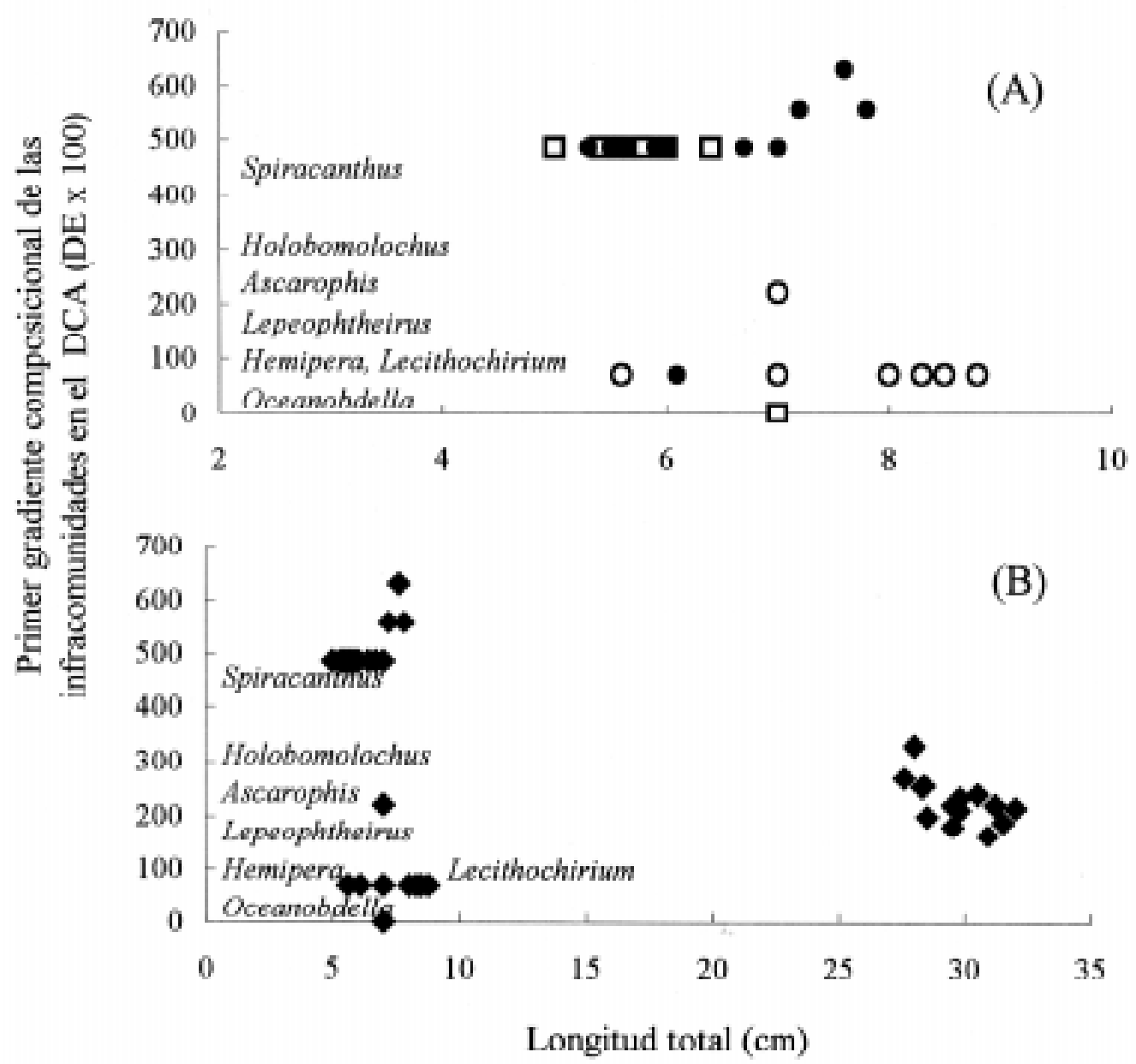

Fig. 2: Relación entre el primer gradiente composicional de las infracomunidades de parásitos (primer eje DCA) y la longitud total de B. chilensis: (A) juveniles (Isla Negra = círculos blancos, Los Bagres = círculos negros, Pucatrihue $=$ cuadrados blancos), y (B) juveniles y adultos de localidades vecinas del litoral centro-sur de Chile $\left(36^{\circ} \mathrm{S}\right)$. Los nombres de los taxa parasitarios se indican frente a su coordenada en el eje.

Relationship between the first compositional gradient of parasite infracommunities (scores on first DCA axis) and host body length of B. chilensis: (A) juveniles, and (B) juveniles and adults from three nearby littoral localities in central-south Chile $\left(\right.$ ca. $36^{\circ} \mathrm{S}$, Isla Negra = white circles, Los Bagres = black circles, Pucatrihue = white squares). Names of parasite taxa are shown in front of their coordinate in that axis. 
Oceanobdella sp., S. bovichthys, y Corynosoma sp. Los seis taxa que eran exclusivos de los adultos en general poseían alta prevalencia y abundancia (Tabla 3). Todos los valores promedio de los descriptores de las infracomunidades de los toritos juveniles eran significativamente menores (o distintos) que los de los adultos de una localidad cercana (prevalencia total (Tablas 4 y 5, Fig. $2 \mathrm{~B})$, intensidad total $\left(\chi^{2}=21,3\right)$, riqueza $\left(\chi^{2}=\right.$ $22,1)$, diversidad $\left(\chi^{2}=22,6\right)$ y composición $\left(\chi^{2}=\right.$ 17,8 , aproximación a Chi-cuadrado de la prueba de Kruskal-Wallis, todos con 1 g.l.; $\mathrm{P}<0,01$ ).

\section{DISCUSIÓN}

Todos los factores ecológicos del huésped, tales como la dieta, gregarismo, densidad de conespecíficos, el uso del hábitat, el tamaño corporal, entre otros, pueden afectar a las infracomunidades de parásitos. Cuando la unidad de estudio es la fauna parasitaria de un linaje diverso de hospedadores en un área, es decir, las comunidades componentes, y la pregunta trata de los factores que afectan la riqueza de especies en las comunidades componentes, también se puede concluir que el tamaño de la especie de hospedador, y la composición de su dieta, pueden ser relevantes para entender sus variaciones. En dichos estudios es usual utilizar una corrección por los efectos de la inercia filogenética (ver Poulin 1998, Morand et al. 2000), pero no son pertinentes a nivel infracomunitario, sino más bien, las ontogenéticas.
Las variaciones geográficas en la dieta de los toritos juveniles (Tabla 2, Fig. 1A) pueden explicar en parte las diferencias parasitarias observadas (Tabla 5), pero no se conocen los ciclos de vida de los parásitos involucrados. Por ejemplo, la menor prevalencia, abundancia y riqueza del parasitismo en los toritos de Burca puede estar asociada a que tenían una dieta distinta a los toritos de las otras localidades (Tabla 2 y 3 , Fig. 1A). Sin embargo, los ejemplares de Isla Negra, Los Bagres y Pucatrihue tenían dietas similares y no tenían parasitofaunas similares. Esto sugiere que una misma especie de hospedador intermediario puede estar o no parasitado con una u otra especie de parásito en distintas localidades. Aunque los datos no fueron analizados en ese sentido, tampoco parece haber una relación clara entre amplitud dietaria y riqueza parasitaria ya que en dos de las tres localidades en que se encontró una gran amplitud dietaria, sólo se encontraron ectoparásitos, los cuales no son transmitidos por vía trófica (Isla Negra y Burca, Tabla 2 y 3). En contraste, las infracomunidades de parásitos encontradas en los toritos juveniles muestreados en Pucatrihue tuvieron mayor riqueza y prevalencia de endoparásitos, a pesar de que su dieta incluía cuatro ítems presa (Tabla 2 y 3 ).

Hay pocos antecedentes de la relación entre la dieta y parásitos en peces del intermareal. Aparentemente, los juveniles presentan grandes variaciones en su parasitofauna, aún cuando se comparan grupos de peces de localidades muy cercanas y que además presentan dietas similares. Algo

TABLA 4

Prevalencia total (\% de hospedadores parasitados), intensidad total (número total de parásitos/ número de hospedadores parasitados), riqueza (número de taxa por hospedador parasitado) y diversidad (índice de Brillouin) de infracomunidades de parásitos metazoos en ejemplares juveniles y adultos de Bovichthys chilensis recolectados de cinco localidades de la costa de Chile; la desviación estándar para cada variable se indica entre paréntesis

Total prevalence (percentage of hosts with parasites), total intensity (total number of parasites / number of parasitized hosts), infracommunity richness (number of parasitic taxa per parasitized host individual) and diversity (Brillouin index) of metazoan parasites in juveniles and adults Bovichthys chilensis from five littoral localities of Chile; standard deviation in each variable is given in parenthesis

\begin{tabular}{|c|c|c|c|c|c|c|c|}
\hline \multirow{2}{*}{ Juvenile } & \multirow[t]{2}{*}{ Localidad } & \multirow[t]{2}{*}{ Prevalencia } & \multicolumn{2}{|c|}{ Intensidad } & \multicolumn{2}{|c|}{ Riqueza } & Diversidad \\
\hline & & & & & & & \\
\hline & Isla Negra & 29,2 & 1,57 & $(0,8)$ & 1,00 & $(0,5)$ & $0,00 \quad(0,0)$ \\
\hline & Burca & 2,9 & 1,00 & $(-)$ & 1,00 & $(-)$ & $0,00 \quad(-)$ \\
\hline & Los Bagres & 39,3 & 4,91 & $(11,6)$ & 1,18 & $(0,4)$ & $0,09 \quad(0,2)$ \\
\hline & Pucatrihue & 71,4 & 2,00 & $(0,9)$ & 1,20 & $(0,4)$ & $0,10 \quad(0,2)$ \\
\hline \multicolumn{8}{|c|}{ Adultos } \\
\hline & Chome & 100 & 37,70 & $(28,8)$ & 4,78 & $(1,4)$ & $1,47 \quad(0,4)$ \\
\hline Total & & 39,3 & 13 & $(22)$ & 2,20 & $(1,9)$ & $0,48 \quad(0,7)$ \\
\hline
\end{tabular}


TABLA 5

Resultado de la comparación de la prevalencia total de parasitismo de los toritos juveniles entre localidades (valor de $\chi^{2}$ con 1 grado de libertad y probabilidad, P), y con los adultos (con la prueba de probabilidad exacta de Fisher)

Results of comparative statistical analyses of the total prevalence of parasitism among juvenile fish from four different localities ( $\chi^{2}$ value with 1 degree of freedom and probability, P), and with adult fish of a $5^{\text {th }}$ locality (with an Fisher's exact probability test)

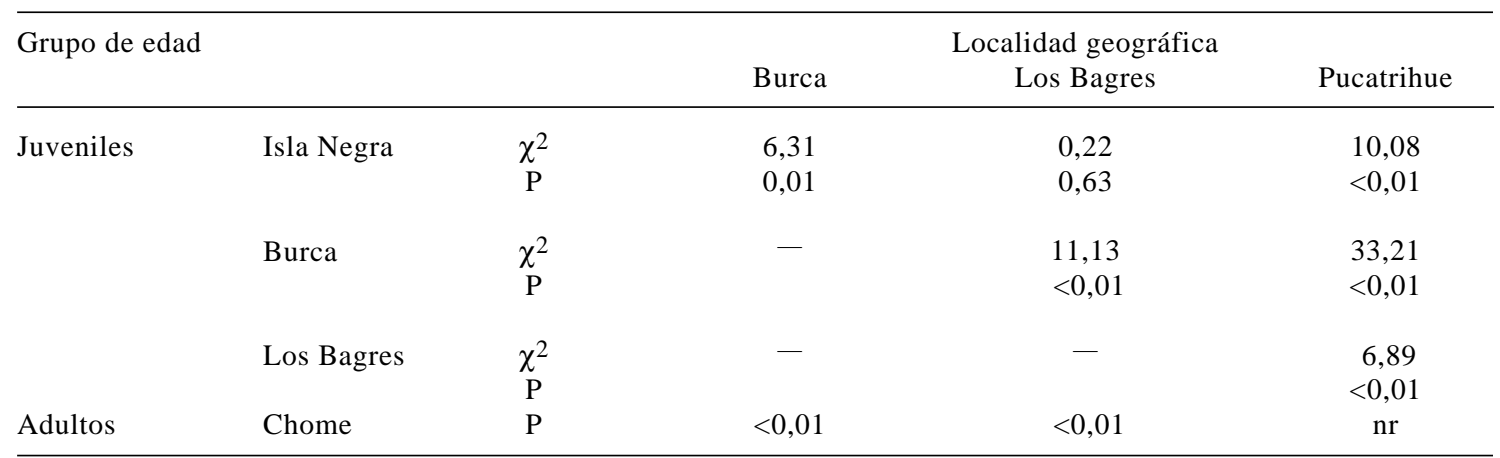

g.l. = 1 para cada una de las comparaciones; $\mathrm{nr}=$ no realizado

así se observó en los juveniles de Notothenia c.f. angustata (Muñoz et al. 2001), quizás porque las parasitosis son recientes debido al corto tiempo de vida que tienen los hospedadores, el cual no habría sido suficiente para que las infracomunidades alcanzaran una configuración "promedio" relativamente estable, característica de esa especie de pez al estado adulto (Muñoz et. al. 2001). Una argumentación similar, pero que no involucra a la dieta, puede ayudar a entender las variaciones geográficas del ectoparasitismo en juveniles.

Un análisis más adecuado que el aquí empleado para abordar la pregunta de la posible existencia de una relación entre la dieta y el endoparasitismo debiera considerar el análisis conjunto de ambos aspectos, y no separadamente, como aquí se hizo. Sin embargo, para evaluar si existe, por ejemplo, una correlación significativa entre la riqueza de taxa en la dieta y la prevalencia del parasitismo se requiere un número mayor de localidades muestreadas, para un mismo estado ontogenético.

Los toritos adultos tenían dieta y parasitofauna distinta a la de los juveniles. En este caso podemos destacar diferencias ecológicas importantes que explicarían las distintas composiciones. Por ejemplo, los toritos adultos habitan en el submareal somero, donde alcanzan mayor longitud total y maduran sexualmente. Con todos estos cambios ontogenéticos, sus hábitos tróficos cambian prácticamente en su totalidad, ya que consumen otro tipo de presas y que son de mayor tamaño que las que consumen los toritos juveniles (Tabla 1 y 2 ). Por lo tanto, el cambio de hábitat y de estado ontogenético conllevan a cambios dietarios, y a subsecuentes cambios en la parasitofauna. Por ejemplo, los crustáceos decápodos, por lo general, son hospedadores intermediarios de Ascarophis (ver Poinar \& Kuris 1975, Poinar \& Thomas 1976, Martorelli et al. 2000), que fue frecuente y abundante sólo en los toritos adultos (Tabla 5).

El tamaño del hospedador es otra variable que afecta la parasitofauna, ya que peces grandes pueden ofrecer mayor espacio físico y variedad de nichos para la colonización de nuevos parásitos. Por lo tanto, los hospedadores grandes pueden albergar a más parásitos, de hecho, los toritos adultos presentaron altas magnitudes de los descriptores parasitológicos (Tabla 3, 4 y 5). Además, las especies parásitas de larga vida pueden acumularse en su hospedador. Hay varios estudios que indican que la presencia de ciertas especies parásitas ocurre en determinados estados ontogenéticos del hospedador (ver Williams \& Jones 1994), en donde los parásitos encuentran las características físico-químicas apropiadas para su desarrollo.

En este estudio encontramos variaciones geográficas importantes en la dieta y parasitismo en los toritos juveniles, sin embargo no sabemos si la variación geográfica es igualmente importante para los adultos. Futuros estudios podrán aclarar si el factor geográfico es o no tan determinante como el ontogenético en la magnitud del cambio en composición de la dieta y de las infracomunidades. Finalmente, cabe considerar que en este estudio, como en pocos otros (Muñoz 
et al. 2001, Aldana et al. 2002), se ha encontrado una escasa y compleja relación cuantitativa entre la composición de la dieta y del parasitismo. Creemos que a pesar de que ambos son procesos biológicamente interrelacionados, es poco probable que se encuentre una correlación entre ambas ya que los meras diferencias en tasas de ingreso (los de los parásitos menores que los de las presas), y tiempos de residencia (los de parásitos mayores que los de las presas) las desdibujarían. Además, la prevalencia total del parasitismo fue baja y variable entre los toritos juveniles, en cambio la presencia de presas fue más frecuente. Esto determinó que había más datos en los que se basó el análisis dietario, el que además era distinto al de las infracomunidades, dificultando aún más la búsqueda de correlaciones entre ambos tipos de variables. Idealmente, tanto la frecuencia de presencia de presas como la de parásitos ha de ser similar y alta en las muestras, y se ha de contar con un mínimo aceptable de lugares de estudio.

\section{AGRADECIMIENTOS}

Agradecemos al Sr. Marco Antonio Retamal (Universidad de Concepción, Chile) por su ayuda en la determinación taxonómica de los crustáceos decápodos. Al Dr. Rodney Bray (The Natural History Museum, London, United Kingdom) por su ayuda en la determinación de los digeneos y monogeneos. Finalmente, agradecemos el financiamiento de los proyectos FONDECYT 1980442 y FONDAP, Programa 3: Ecología y Conservación.

\section{LITERATURA CITADA}

ALDANA M, JM PULGAR, F OGALDE \& FP OJEDA (2002) Morphometric and parasitological evidence for ontogenetic and geographical shifts in trophic status of intertidal fishes. Bulletin of Marine Science 70: 55-74.

BUSH AO, KD LAFFERTY, JM LOTZ \& AW SHOSTACK (1997) Parasitology meets ecology on its own terms: Margolis et al. revisited. Journal of Parasitology 83: 575-583.

FARIÑA JM, M ALDANA F OGALDE \& FP OJEDA (2000) Ecología trófica de Girella laevifrons (Pisces: Kyphosidae) en zonas intermareales rocosas del norte de Chile afectadas y no afectadas por contaminantes derivados de la minería del cobre. Revista Chilena de Historia Natural 73: 139-149.

HYSLOP EJ (1980) Stomach contents analysis: a review of methods and their application. Journal of Fish Biology 17: 411-429.
JONGMAN RHG, CJF TER BRAAK \& OFR VAN TONGEREN (1995) Data analysis in community and landscape ecology. Cambridge University Press, New York, New York. 299 pp.

KABATA Z (1979) Parasitic Copepoda of British fishes. The Ray Society, The British Museum, London, United Kingdom. 468 pp.

KENNEDY CR (1975) Ecological animal parasitology. Blackwell Scientific Publications, Boston, Massachusetts. 163pp.

MAGURRAN AE (1988) Ecological diversity and its measurements. Princeton University Press, Princeton, New Jersey. 175 pp.

MANN G (1954) Vida de los peces de aguas chilenas: clave para los peces chilenos. Ministerio de Agricultura, Santiago, Chile. 342pp.

MARTORELLI SR, GT NAVONE \& V IVANOV (2000) Proposed life cycle of Ascarophis marina (Nematoda: Cystidicolidae) in Argentine waters. Journal of Parasitology 86: 1047-1050.

MORAND S, TH CRIBB, M KULBICKI, MC RIGBY, C CHAUVET, V DUFOUR, E FALIEX, R GALZIN, CM LO, A LO-YAT, S PICHELIN \& P SASAL (2000) Endoparasite species richness of New Caledonian butterfly fishes: host density and diet matter. Parasitology 121: 65-73

MORENO C \& JC CASTILLA (1975) Guía para el reconocimiento y observación de peces de Chile. Editora Nacional Gabriela Mistral, Santiago, Chile. 120pp.

MUÑOZ AA \& FP OJEDA (1997) Feeding guild structure of a rocky intertidal fish assemblage in central Chile. Environmental Biology of Fishes 49: 471-479.

MUÑOZ AA \& FP OJEDA (1998) Guild structure of carnivorous intertidal fishes of the Chilean coast: implications of ontogenetic dietary shifts. Oecologia 114: 563-573.

MUÑOZ AA \& FP OJEDA (2000) Ontogenetic changes in the diet of the herbivorous fish Scartichthys viridis in a rocky intertidal zone in central Chile. Journal of Fish Biology 56: 986-998.

MUÑOZ G, F GARCÍAS, V VALDEBENITO \& M GEORGE-NASCIMENTO (2001) Parasitofauna y alimentación de Notothenia c.f. angustata Hutton, 1875 (Pisces: Nototheniidae) del intermareal de dos localidades del Golfo de Arauco, Chile. Boletín Chileno de Parasitología (Chile) 56: 29-33.

MUÑOZ G \& M GEORGE-NASCIMENTO (2002) Spiracanthus bovichthys n. gen. n. sp. (Acanthocephala: Arhythmacanthidae), a parasite of littoral fishes of the central-south coast of Chile. Journal of Parasitology 88: 141-145.

OJEDA FP \& AA MUÑOZ (1999) Feeding selectivity of the herbivorouos fish Scartichthys viridis: effects on macroalgal community structure in a temperate rocky intertidal coastal zone. Marine Ecology Progress Series 184: 219-229.

PIELOU EC (1984) The interpretation of ecological data. John Wiley \& Sons, New York, New York. 263 pp.

POINAR GO \& AM KURIS (1975) Juvenile Ascarophis (Spirurida: Nematoda) parasitizing intertidal decapod crustacean in California, with notes on prevalence and effect on host growth and survival. Journal of Invertebrate Pathology 26: 375-382. 
POINAR GO \& GM THOMAS (1976) Occurrence of Ascarophis (Spirurida: Nematoda) on Callianasa californiensis Dana and other decapod crustaceans. Proceedings of the Helminthological Society of Washington 43: 28-33.

POULIN R (1998) Evolutionary ecology of parasites: from individuals to communities. Chapman \& Hall, London, United Kingdom. 212 pp.

QUIJADA P \& C CÁCERES (2000) Patrones de abundancia, composición trófica y distribución espacial del ensamble de peces intermareales de la zona centrosur de Chile. Revista Chilena de Historia Natural 73: 739-747.

RIFFO R \& M GEORGE-NASCIMENTO (1992) Variaciones de la abundancia de larvas de Anisakis sp. e Hysterothylacium sp. (Nematoda: Anisakidae) en la merluza de cola Macruronus magellanicus Lönnberg 1862: la importancia del sexo, tamaño corporal y dieta del hospedador. Estudios Oceanológicos (Chi1e) $11: 79-84$

ROHDE K (1984) Ecology of marine parasites. Helgoländer Meeresuntersuchungen 37: 5-33.

SIEGEL S \& NJ CASTELLAN (1988) Nonparametric statistics for the behavioral sciences. Second edition, McGraw-Hill Book Company, New York, New York. $399 \mathrm{pp}$.

Editor Asociado: P. Ojeda

Recibido el 11 de abril de 2002; aceptado el 12 de agosto de 2002
VALTONEN T \& M JULKUNEN (1995) Influence of the transmission of parasites from prey fishes on the composition of the parasite community of a predatory fish. Canadian Journal of Fisheries and Aquatic Sciences 52: 233-245.

WILLIAMS H \& A JONES (1994) Parasitic worms of fish. Taylor \& Francis Ltd., London, United Kingdom. 593 pp.

YAMAGUTI S (1971) Synopsis of digenetic trematodes of vertebrates. Keigaku Publishing Co., Tokyo, Japan. $474 \mathrm{pp}$.

ZANDER CD, LW REIMER \& K BARZ (1999) Parasite communities of the Salzhaff (Northwest Mecklenburg, Baltic Sea). I. Structure and dynamics of communities of littoral fish, especially small-sized fish. Parasitology Research 85: 356-372.

ZAR JH (1996) Biostatistical analysis. Second edition. Prentice-Hall Inc., Englewood Cliffs, New Jersey. $622 \mathrm{pp}$.

ZDZITOWIECKI K (1991) Antarctic Acanthocephala. Synopses of the Antarctic Benthos. Koeltz Scientific Books, Königstein, Germany 3: 1-116. 